\title{
ORIGINAL
}

\section{CARACTERÍSTICAS DE LAS URGENCIAS HOSPITALARIAS DEL SERVICIO ANDALUZ DE SALUD DURANTE 2012 A PARTIR DEL CONJUNTO MÍNIMO BÁSICO DE DATOS DE URGENCIAS}

\author{
Juan Antonio Goicoechea Salazar (1), María Adoración Nieto García (2), Antonio Laguna \\ Téllez (1), Daniel Larrocha Mata (1), Vicente David Canto Casasola (1) y Francisco Murillo \\ Cabezas (3).
}

(1) Servicio de Producto Sanitario. Servicio Andaluz de Salud. Sevilla. España.

(2) Departamento de Medicina Preventiva y Salud Pública. Facultad de Medicina de la Universidad de Sevilla. España.

(3) Departamento de Medicina. Facultad de Medicina de la Universidad de Sevilla. España.

No existe ningún conflicto de intereses.

Este trabajo se ha llevado a cabo sin financiación externa

\section{RESUMEN}

Fundamentos: La implantación de Diraya-Urgencias en los hospitales del Servicio Andaluz de Salud (SAS) y el desarrollo de un codificador automático propio ha permitido instaurar el Conjunto Mínimo Básico de Datos de Urgencias (CMBD-U). El objetivo de este artículo es describir la casuística de los servicios de urgencias hospitalarios utilizando las distintas dimensiones contenidas en el CMBD-U.

Métodos: Utilizando el CMBD-U, se clasificaron 3.235.600 registros de urgencias hospitalarias de 2012 en categorías clínicas mediante el código CIE-9-MC proporcionado por el codificador automático. Se definieron reglas de validez para la explotación de los tiempos. Se realizó un análisis descriptivo obteniendo indicadores demográficos, cronológicos, tasas de hospitalización, retorno y exitus y tiempos asistenciales y de permanencia en urgencias.

Resultados: Las mujeres generaron el $54,26 \%$ de las urgencias. Su edad media (39,98 años) superó a la de los hombres (37,61). El 21,49\% fueron urgencias pediátricas. La máxima afluencia horaria fue de 10:00 a 13:00 y de 16:00 a 17:00. Los pacientes que no pasaron por observación $(92,67 \%)$ permanecieron en urgencias 153 minutos de media. Más del $50 \%$ de las urgencias fueron generadas por lesiones e intoxicaciones, enfermedades respiratorias, osteomusculares y síntomas y signos. Entre los procesos asistenciales integrados se identificaron $79.191 \mathrm{ca}$ sos de dolor torácico, 28.741 de insuficiencia cardiaca y 27.989 infecciones graves.

Conclusiones: El CMBD-U permite analizar sistemáticamente las urgencias hospitalarias identificando la actividad desarrollada, la casuística atendida, los tiempos de respuesta asistencial y permanencia en urgencias y la calidad asistencial.

Palabras clave: Servicios Médicos de urgencia. Case-mix. Indicadores de calidad de la atención de salud. Sistemas de información en salud. Codificación clínica.

\section{Correspondencia}

Juan Antonio Goicoechea Salazar

Servicio Andaluz de Salud.

Avenida de la Constitución, 18

41071 Sevilla

jantonio.goicoechea@juntadeandalucia.es

\section{ABSTRACT \\ Case-Mix of Hospital Emergencies in the Andalusian Health Service Based on the 2012 Minimum Data Set. Spain}

Background: The implementation of digital health records in emergency departments (ED) in hospitals in the Andalusian Health Service and the development of an automatic encoder for this area have allowed us to establish a Minimum Data Set for Emergencies (MDS-ED). The aim of this article is to describe the case mix of hospital EDs using various dimensions contained in the MDS-ED.

Methods: 3.235 .600 hospital emergency records in 2012 were classified in clinical categories from the ICD-9-CM codes generated by the automatic encoder. Operating rules to obtain response time and length of stay were defined. A descriptive analysis was carried out to obtain demographic and chronological indicators as well as hospitalization, return and death rates and response time and length of stay in the EDs.

Results: Women generated $54,26 \%$ of all occurrences and their average age $(39,98$ years) was higher than men's $(37,61)$. Paediatric emergencies accounted for $21,49 \%$ of the total. The peak hours were from 10:00 to 13:00 and from 16:00 to 17:00. Patients who did not undergo observation $(92,67 \%)$ remained in the ED an average of 153 minutes. Injuries and poisoning, respiratory diseases, musculoskeletal diseases and symptoms and signs generated over $50 \%$ of all visits. 79.191 cases of chest pain, 28.741 episodes of heart failure and 27.989 episodes of serious infections were identified among the most relevant disorders.

Conclusions: The MDS-ED makes it possible to address systematically the analysis of hospital emergencies by identifying the activity developed, the case-mix attended, the response times, the time spent in ED and the quality of the care.

Key words: Emergency medical services. Case-mix. Quality indicators, health care. Health information systems. Clinical coding. 


\section{INTRODUCCIÓN}

La demanda de atención urgente en los hospitales del Servicio Andaluz de Salud (SAS) ha mostrado una tendencia creciente en las dos últimas décadas, con un incremento medio de 100.000 urgencias anua$\operatorname{les}^{1-3}$. En 2010 se produjeron en Andalucía del orden de 600 asistencias hospitalarias urgentes por cada 1.000 habitantes $^{4}$. En 2011 las urgencias no ingresadas representaron el $8,41 \%$ del gasto hospitalario del $\mathrm{SAS}^{5}$. El mismo año, las urgencias fueron la puerta de entrada del $60 \%$ de los episodios de hospitalización ${ }^{3}$, ámbito que generó el $45,10 \%$ del gasto hospitalario ${ }^{5}$.

Aunque la gestión de la asistencia urgente es uno de los grandes retos a que se enfrentan los servicios sanitarios, son pocos los estudios llevados a cabo en España sobre la casuística atendida en los servicios de urgencias hospitalarios dada la dificultad que, por su volumen, presenta la codificación de sus episodios.

La implantación en las urgencias hospitalarias del SAS de la aplicación DirayaUrgencias ${ }^{6}$ ha permitido disponer en soporte informático de los datos clínicos y administrativos de los episodios atendidos. No obstante, la mayor parte de la información clínica se registra en lenguaje natural, lo que dificulta enormemente su tratamiento y su utilización para objetivos distintos a los estrictamente asistenciales ${ }^{7}$.

Con el fin de abordar esta situación y configurar el Conjunto Mínimo Básico de Datos de Urgencias (CMBD-U) del Sistema Sanitario Público de Andalucía (SSPA) se desarrolló un codificador automático para asignar códigos CIE-9-MC a los textos diagnósticos recogidos en la historia digital de urgencias. Su estructura y evaluación están en trámite de publicación.

El objetivo de este artículo es analizar la casuística de los Servicios de Urgencias hospitalarios del SAS y estudiar sus episodios en función de las patologías atendidas, la edad y sexo de los pacientes, los tiempos de respuesta asistencial y de permanencia en urgencias y su distribución temporal (horaria, diaria y mensual).

Este estudio es el primero llevado a cabo en España sobre el total de las urgencias atendidas por un Servicio Regional de Salud, que en el caso del SAS da cobertura a 6 millones y medio de andaluces. Muestra resultados para todo el universo de análisis con base en los registros administrativos del CMBD-U permitiendo superar las limitaciones de los estudios llevados a cabo hasta la fecha, que abarcan ámbitos asistenciales o periodos temporales restringidos ${ }^{8-15}$.

\section{MATERIAL Y MÉTODOS}

Fuente de información: CMBD-U. Se analizaron 3.235.600 registros de urgencias de 28 hospitales del SAS en 2012 conteniendo variables clínicas y administrativas y textos diagnósticos escritos por los facultativos en la historia de salud digital. No se incluyeron los registros en que se desconocía la edad y/o sexo de los pacientes $(0,29 \%)$.

Codificación de textos diagnósticos. Se utilizó un codificador automático propio basado en reglas de procesamiento del lenguaje natural y en el uso de diccionarios para asignar uno o más códigos CIE-9-MC a cada texto clínico y los correspondientes códigos de causas externas (códigos E). El codificador ordenó los códigos asignados, colocando primero los procedentes de textos incorporados al informe de alta, así como los más específicos.

Identificación de dimensiones clínicas. Dos documentalistas identificaron los códigos CIE-9-MC de los Procesos Asistenciales Integrados (PAI) priorizados por el Plan Andaluz de Urgencias y Emergencias ${ }^{16,17}$. Se identificaron los PAI a los que corres- 
pondía cada registro teniendo en cuenta todos sus códigos diagnósticos.

Por otra parte, cada registro también fue asignado a una categoría y a un grupo clínico a partir del primer código CIE-9-MC proporcionado por el codificador automático, utilizando el Clinical Classification Software $(\mathrm{CCS})^{18}$. Se calculó el porcentaje de urgencias por categoría CCS sin incluir las urgencias no clasificadas.

Cálculo de tiempos. Se obtuvieron el intervalo horario, el día de la semana, el mes, trimestre y año de cada admisión y alta. Se calculó el tiempo desde la admisión hasta la primera anotación médica en la historia clínica como aproximación al tiempo transcurrido hasta la primera asistencia médica en consulta (TPAM). Se calculó el tiempo de permanencia en urgencias (TPU) como el transcurrido desde la admisión hasta la salida del servicio tras el alta. Los TPU se promediaron para el total de pacientes y para los que no pasaron por observación.

Se calcularon los porcentajes de pacientes: 1) que sin pasar por observación permanecieron más de 4 horas en urgencias y 2) que pasando por observación permanecieron más de 24 horas.

Dada la distribución asimétrica del TPAM y del TPU y la existencia de algunas observaciones anómalas, para el análisis de estos tiempos se extrajeron los registros en que cualquiera de ellos era negativo o mayor que 3.360 minutos. Este fue el punto de corte superior obtenido al aplicar la caja rápida de Tukey a los TPU de los pacientes que pasando por observación fueron ingresados, ya que estos tuvieron los mayores TPU. Ello afectó al 1,05\% de los registros. Igualmente, a efectos del cálculo de estos tiempos, no se tuvieron en cuenta 91 registros sin fecha de alta.

El porcentaje de ingresos en planta se calculó excluyendo los registros de un hospital
$(9,61 \%)$ que presentaba un comportamiento muy diferente al resto.

Identificación etaria. Se identificó la edad de los pacientes (días, meses y años) y sus grupos etarios de pertenencia (quinquenio, decenio, otros). Los pacientes menores de 15 años fueron clasificados como pediátricos.

Identificación de retornos. Se identificaron los registros de pacientes que habían solicitado una nueva asistencia urgente en menos de 72 horas.

\section{RESULTADOS}

Como se desprende de las tablas 1 y 2 , el $54,26 \%$ de las urgencias fueron de mujeres $(56,72 \%$ en personas adultas, $45,27 \%$ en pediatría) y un $21,49 \%$ pediátricas. La edad media fue de 38,90 años (48,29 adultos, 4,57 pediatría). El 9,25\% requirieron ingreso $(10,67 \%$ adultos, $3,71 \%$ pediatría $)$. El porcentaje de retornos fue $8,69 \%(8,64 \%$ adultos, $8,83 \%$ pediatría). La mortalidad global fue $0,12 \%(0,15 \%$ adultos, menos del $0,01 \%$ en pediatría).

El TPU medio fue de 203 minutos (225 adultos y 123 pediatría). El tiempo medio transcurrido hasta que los pacientes fueron atendidos en consulta médica fue de 57 minutos (60 adultos y 46 pediatría).

El 59,17\% de las urgencias por enfermedades infecciosas y el 52,25\% de las respiratorias fueron pediátricas.

La tabla 1 presenta las urgencias en adultos por categorías CCS. Cinco categorías acumularon prácticamente el $60 \%$ de las urgencias codificadas: Lesiones e intoxicaciones, enfermedades osteomusculares, de los órganos de los sentidos, genitourinarias y síntomas y signos.

Seis categorías, que representan el $26,44 \%$ de las urgencias codificadas, pre- 
Tabla 1

Indicadores de urgencias en adultos (total y por categorías clínicas CCS). 28 Hospitales del SAS 2012

\begin{tabular}{|c|c|c|c|c|c|c|c|c|c|c|c|c|}
\hline CATEGORIAS CCS & urgencias & $\begin{array}{c}\% \text { sobre } \\
\text { clasificadas }\end{array}$ & $\begin{array}{c}\% \\
\text { mujeres }\end{array}$ & $\begin{array}{l}\text { edad } \\
\text { media }\end{array}$ & $\begin{array}{c}\% \\
\text { ingresos }\end{array}$ & $\begin{array}{c}\% \\
\text { retornos } \\
72 \mathrm{~h} .\end{array}$ & $\begin{array}{c}\% \\
\text { exitus }\end{array}$ & TPU & $\begin{array}{c}\text { TPU } \\
\text { sin } \\
\text { observación } \\
\dagger\end{array}$ & $\begin{array}{c}\% \text { TPU }>4 \mathrm{~h} \\
\sin \\
\text { observación } \\
\ddagger\end{array}$ & $\begin{array}{c}\% \text { TPU }>24 \mathrm{~h} \\
\text { con } \\
\text { observación } \\
\S\end{array}$ & TPAM $\|$ \\
\hline Sin clasificar & 362.858 & & 57,06 & 47,87 & 12,66 & 12,40 & 0,05 & 225 & 192 & 18,82 & 17,09 & 96 \\
\hline 01 - Enfermedad Infecciosa y parasitaria & 28.398 & 1,30 & 59,58 & 41,99 & 10,63 & 9,11 & 0,72 & 227 & 143 & 14,48 & 32,85 & 55 \\
\hline 02 - Neoplasias & 16.111 & 0,74 & 48,18 & 59,90 & 34,89 & 10,69 & 1,13 & 420 & 248 & 34,25 & 22,96 & 51 \\
\hline 04 - Enfermedades de la sangre y órganos hematopoyéticos & 17.946 & 0,82 & 57,19 & 67,48 & 18,94 & 5,70 & 0,22 & 610 & 364 & 52,64 & 17,84 & 52 \\
\hline 05 - Enfermedades Mentales & 73.934 & 3,40 & 55,89 & 44,21 & 10,06 & 10,61 & 0,04 & 218 & 176 & 20,10 & 14,95 & 48 \\
\hline 06 - Enfermedades del sistema nervioso y órganos sentidos & 188.783 & 8,67 & 56,16 & 49,84 & 2,88 & 9,83 & 0,03 & 152 & 124 & 11,35 & 17,08 & 55 \\
\hline 07 - Enfermedades del aparato circulatorio & 178.724 & 8,21 & 51,20 & 63,77 & 24,64 & 6,36 & 0,54 & 442 & 263 & 38,09 & 23,29 & 45 \\
\hline 08 - Enfermedades del aparato respiratorio & 163.205 & 7,50 & 48,39 & 57,06 & 18,77 & 7,19 & 0,39 & 324 & 209 & 28,28 & 22,95 & 50 \\
\hline 09 - Enfermedades del aparato digestivo & 175.499 & 8,06 & 52,20 & 50,23 & 18,20 & 9,49 & 0,13 & 324 & 215 & 30,37 & 23,06 & 51 \\
\hline 10 - Enfermedades del sistema genitourinario & 186.091 & 8,55 & 64,03 & 47,17 & 6,63 & 9,90 & 0,06 & 246 & 186 & 23,71 & 24,76 & 53 \\
\hline 11 - Complicaciones del embarazo, parto y puerperio & 131.929 & 6,06 & 99,43 & 30,15 & 29,85 & 14,52 & - & 140 & 130 & 8,97 & 12,65 & 71 \\
\hline 12 - Enfermedades de la piel y tejido subcutáneo & 35.804 & 1,64 & 50,30 & 47,41 & 7,18 & 10,85 & 0,02 & 166 & 137 & 14,32 & 23,29 & 57 \\
\hline 13 - Enfermedades del sistema osteomuscular y conectivo & 265.083 & 12,17 & 56,52 & 47,93 & 0,78 & 5,03 & - & 124 & 117 & 8,72 & 15,22 & 54 \\
\hline 14 - Anomalías Congénitas & 801 & 0,04 & 57,55 & 45,95 & 8,10 & 10,49 & - & 226 & 153 & 18,02 & 31,25 & 56 \\
\hline 15 - Determinadas afecciones originadas en período perinatal & 432 & 0,02 & 57,18 & 33,50 & 3,69 & 6,94 & - & 128 & 114 & 8,75 & 25,00 & 58 \\
\hline 16 - Lesiones e intoxicaciones & 430.983 & 19,79 & 46,97 & 44,89 & 5,15 & 4,89 & 0,03 & 149 & 126 & 8,91 & 19,06 & 50 \\
\hline 17 - Síntomas, signos, condiciones mal definidas y factores que influyen en el estado de salud & 186.058 & 8,54 & 60,30 & 46,62 & 4,34 & 9,64 & 0,05 & 218 & 184 & 25,37 & 14,46 & 53 \\
\hline 18 - Códigos residuales, no clasificados y todos los códigos E 259 y 260 & 73.501 & 3,38 & 60,78 & 40,91 & 1,35 & 9,56 & 1,11 & 182 & 172 & 20,39 & 12,37 & 105 \\
\hline Total adultos & 2.540 .310 & 100,00 & 56,72 & 48,29 & 10,67 & 8,64 & 0,15 & 225 & 168 & 18,37 & 21,21 & 60 \\
\hline
\end{tabular}

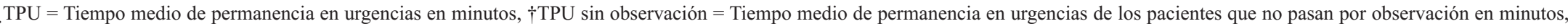

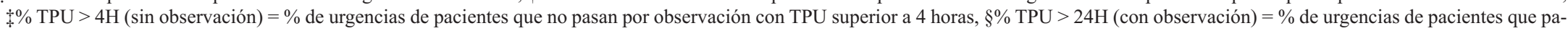
san por observación con TPU superior a 24 horas, $\|$ TPAM= Tiempo medio hasta la primera asistencia médica en minutos 
sentaron una edad media, un porcentaje de ingreso y un TPU elevados, encontrándose sus TPAM entre los más bajos: neoplasias, enfermedades endocrinas e inmunitarias, enfermedades de la sangre, circulatorias, respiratorias y digestivas.

Las complicaciones del embarazo también presentaron un alto porcentaje de ingreso $(29,85 \%)$ aunque su edad media fue la menor (30,15 años).

En todas las categorías predominaron las mujeres excepto en las enfermedades respiratorias $(48,39 \%)$, neoplasias $(48,18 \%)$, lesiones e intoxicaciones $(46,97 \%)$ y enfermedades endocrinas e inmunitarias (46,32\%).

Cinco categorías tuvieron porcentajes de retorno superiores al 10\%: complicaciones del embarazo (14,52\%), enfermedades de la piel $(10,85 \%)$, neoplasias $(10,69 \%)$ enfermedades mentales $(10,61 \%)$ y anomalías congénitas $(10,49 \%)$. Las categorías con menor porcentaje de retorno fueron las enfermedades osteomusculares $(5,03 \%)$ y lesiones e intoxicaciones $(4,89 \%)$.

En cuatro categorías los fallecimientos superaron el $0,5 \%$ : neoplasias $(1,13 \%)$, códigos residuales $(1,11 \%)$, enfermedades infecciosas $(0,72 \%)$ y enfermedades circulatorias $(0,54 \%)$.

Las enfermedades osteomusculares, las complicaciones del embarazo y las lesiones e intoxicaciones tuvieron un TPU medio inferior a 150 minutos (124, 140 y 149 respectivamente). Destacó el alto TPAM medio en la categoría de códigos residuales (105 minutos).

La tabla 2 presenta las urgencias pediátricas por categorías CCS. Las enfermedades respiratorias, lesiones e intoxicaciones, síntomas y signos, y enfermedades digestivas generaron más del 75\% de las urgencias codificadas $(28,47 \%, 20,84 \%, 14,18 \%$ y $11,77 \%$ respectivamente)
Las enfermedades endocrinas e inmunitarias y las de la sangre tuvieron los mayores porcentajes de ingreso $(37,93 \%$ y $33,17 \%$ respectivamente) y los mayores TPU medios (353 y 276 minutos respectivamente) aunque sus TPAM fueron de los mas bajos (34 y 37 minutos respectivamente)

Al contrario que en personas adultas, en la mayoría de las categorías predominaron los hombres. Las enfermedades mentales y las genitourinarias se encontraron entre las excepciones con el $45,58 \%$ y el $45,06 \%$ respectivamente.

La menor edad media se dio en las afecciones de origen perinatal $(0,12$ años $)$ y fue menor de 3 años en las enfermedades infecciosas y respiratorias $(2,95$ y 2,68 años respectivamente). La edad media superó los 8 años en las enfermedades mentales, circulatorias y osteomusculares $(9,49 ; 8,52$ y 8,16 años respectivamente).

En las afecciones de origen perinatal y en las enfermedades de la sangre los retornos superaron el 12\% (13,56\% y $12,81 \%$ respectivamente). Los fallecimientos superaron el $0,05 \%$ en las enfermedades circulatorias $(0,16 \%)$ y en las endocrinas e inmunitarias $(0,05 \%)$.

En 4 categorías el TPU medio fue inferior a 100 minutos: Enfermedades de los órganos de los sentidos (99), osteomusculares (96), lesiones e intoxicaciones (94) y enfermedades de la piel (72).

El menor TPAM se dio en las afecciones de origen perinatal (30 minutos) y el mayor, como ocurrió en adultos, en los códigos residuales (117).

Como muestra la tabla 3 , se identificaron 79.191 casos de dolor torácico, 28.741 insuficiencias cardiacas, 27.989 infecciones graves, 21.247 accidentes cerebrales vasculares (ACV), 17.737 
Tabla 2

Indicadores de urgencias pediátricas (total y por categorías clínicas CCS). 28 Hospitales del SAS 2012

\begin{tabular}{|c|c|c|c|c|c|c|c|c|c|c|c|c|}
\hline CATEGORIAS CCS & urgencias & $\begin{array}{c}\% \text { sobre } \\
\text { clasificadas }\end{array}$ & $\begin{array}{c}\% \\
\text { mujeres }\end{array}$ & $\begin{array}{c}\text { edad } \\
\text { media }\end{array}$ & \begin{tabular}{|c|}
$\%$ \\
ingresos
\end{tabular} & $\begin{array}{c}\% \\
\text { retornos } \\
72 \mathrm{~h}\end{array}$ & $\begin{array}{c}\% \\
\text { exitus }\end{array}$ & TPU & $\begin{array}{c}\text { TPU sin } \\
\text { observación } \\
\dagger\end{array}$ & $\begin{array}{c}\% \text { TPU }>4 h \\
\sin \\
\text { observación } \\
\ddagger\end{array}$ & \begin{tabular}{|c|}
$\%$ TPU $>$ \\
$24 \mathrm{~h}$ con \\
observación \\
$\S$
\end{tabular} & TPAM ॥ \\
\hline Sin clasificar & 68.101 & & 44,50 & 5,12 & 5,12 & 12,26 & 0,01 & 173 & 156 & 11,15 & 7,22 & 99 \\
\hline 01 - Enfermedad Infecciosa y parasitaria & 41.156 & 6,56 & 46,74 & 2,95 & 1,44 & 11,32 & - & 113 & 93 & 5,15 & 5,51 & 39 \\
\hline 02 - Neoplasias & 531 & 0,08 & 49,53 & 5,90 & 16,48 & 9,79 & - & 134 & 120 & 7,16 & - & 38 \\
\hline 03 - Enfermedades endocrinas, nutricionales y metabólicas y trastornos de inmunidad & 1.947 & 0,31 & 49,82 & 5,24 & 37,93 & 10,17 & 0,05 & 353 & 232 & 21,96 & 14,19 & 34 \\
\hline 04 - Enfermedades de la sangre y órganos hematopoyéticos & 1.179 & 0,19 & 43,43 & 5,88 & 33,17 & 12,81 & - & 276 & 224 & 21,62 & 11,26 & 37 \\
\hline 05 - Enfermedades Mentales & 1.957 & 0,31 & 54,42 & 9,49 & 6,72 & 8,53 & - & 158 & 124 & 9,60 & 6,88 & 39 \\
\hline 06 - Enfermedades del sistema nervioso y órganos sentidos & 48.532 & 7,74 & 46,54 & 4,72 & 4,70 & 9,70 & - & 99 & 79 & 3,35 & 6,72 & 38 \\
\hline 07 - Enfermedades del aparato circulatorio & 3.132 & 0,50 & 43,33 & 8,52 & 9,59 & 5,91 & 0,16 & 157 & 129 & 8,50 & 4,48 & 39 \\
\hline 08 - Enfermedades del aparato respiratorio & 178.587 & 28,47 & 44,56 & 2,68 & 3,22 & 9,30 & - & 117 & 95 & 4,50 & 6,98 & 40 \\
\hline 09 - Enfermedades del aparato digestivo & 73.804 & 11,77 & 45,91 & 3,68 & 5,10 & 11,79 & - & 147 & 110 & 6,71 & 8,50 & 37 \\
\hline 10 - Enfermedades del sistema genitourinario & 15.369 & 2,45 & 54,94 & 5,24 & 8,68 & 8,86 & - & 151 & 133 & 10,54 & 5,39 & 38 \\
\hline 11 - Complicaciones del embarazo, parto y puerperio & 211 & 0,03 & 81,04 & 8,93 & 15,43 & 11,37 & - & 129 & 99 & 3,59 & 6,67 & 45 \\
\hline 12 - Enfermedades de la piel y tejido subcutáneo & 8.888 & 1,42 & 47,49 & 4,54 & 2,85 & 8,49 & - & 72 & 65 & 1,88 & 3,85 & 37 \\
\hline 13 - Enfermedades del sistema osteomuscular y conectivo & 21.006 & 3,35 & 46,40 & 8,16 & 2,24 & 6,15 & - & 96 & 91 & 3,63 & 9,36 & 43 \\
\hline 14 - Anomalías Congénitas & 602 & 0,10 & 41,86 & 3,16 & 8,96 & 9,47 & - & 135 & 113 & 7,95 & 6,45 & 40 \\
\hline 15 - Determinadas afecciones originadas en período perinatal & 1.748 & 0,28 & 42,28 & 0,12 & 9,90 & 13,56 & - & 131 & 123 & 8,02 & 0,90 & 30 \\
\hline 16 - Lesiones e intoxicaciones & 130.703 & 20,84 & 41,99 & 7,25 & 1,78 & 4,25 & - & 94 & 86 & 2,55 & 3,75 & 42 \\
\hline 17 - Síntomas, signos, condiciones mal definidas y factores que influyen en el estado de salud & 88.925 & 14,18 & 47,64 & 4,28 & 3,93 & 8,66 & - & 133 & 109 & 7,23 & 7,03 & 36 \\
\hline 18 - Códigos residuales, no clasificados y todos los códigos E 259 y 260 & 8.912 & 1,42 & 46,68 & 3,98 & 1,03 & 7,39 & 0,02 & 176 & 173 & 15,43 & 3,36 & 117 \\
\hline Total pediatría & 695.290 & 100,00 & 45,27 & 4,57 & 3,71 & 8,83 & - & 123 & 103 & 5,61 & 6,94 & 46 \\
\hline
\end{tabular}

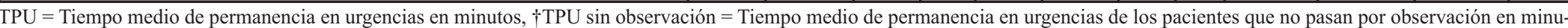

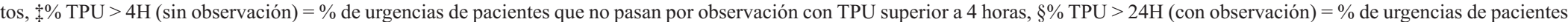
que pasan por observación con TPU superior a 24 horas, $\|$ TPAM= Tiempo medio hasta la primera asistencia médica en minutos 
Tabla 3

Procesos asistenciales integrados (PAIs) identificados en el CMBD de Urgencias. 28 Hospitales del SAS 2012

\begin{tabular}{|c|c|c|c|}
\hline PROCESO & NÚMERO & GRUPO PAI & NÚMERO \\
\hline \multirow{4}{*}{ Dolor torácico } & \multirow{4}{*}{79.191} & 01 Cardiopatía isquémica & 17.946 \\
\hline & & 02 Tromboembolismo pulmonar & 2.097 \\
\hline & & 03 Síndrome aórtico agudo & 332 \\
\hline & & 04 Dolor torácico genérico & 58.816 \\
\hline $\mathrm{ACV}$ & 21.247 & $05 \mathrm{ACV}$ & 21.247 \\
\hline EPOC & 17.737 & 06 EPOC & 17.737 \\
\hline Insuficiencia cardiaca & 28.741 & 07 Insuficiencia cardiaca & 28.741 \\
\hline \multirow{11}{*}{ Trauma grave } & \multirow{11}{*}{18.940} & 8 Lesiones de órganos internos y vasculares & - \\
\hline & & 09 Amputación dedos manos & 340 \\
\hline & & 10 Amputación dedos pies & 10 \\
\hline & & 11 Amputación proximal muñeca o tobillo & 3 \\
\hline & & 12 Tórax Basculante & 9 \\
\hline & & 13 Fractura húmero o fémur & 16.373 \\
\hline & & 14 Fractura bóveda craneal & 491 \\
\hline & & 15 Fractura de pelvis & 1.714 \\
\hline & & 16 Quemaduras $>10 \%$ corporal & \\
\hline & & 17 Fractura con afectación vascular & \\
\hline & & 18 Lesión medular & \\
\hline Fractura cadera ancianos & 6.186 & 19 Fractura cadera ancianos & 6.186 \\
\hline \multirow{5}{*}{ Infecciones graves } & \multirow{5}{*}{27.989} & 20 SIRS & 3.202 \\
\hline & & 21 Meningitis & 750 \\
\hline & & 22 Encefalitis & 188 \\
\hline & & 23 Neumonía & 23.773 \\
\hline & & 24 Septicemia & 76 \\
\hline Total & 200.031 & & 200.031 \\
\hline
\end{tabular}

enfermedades pulmonares obstructivas crónicas (EPOC), 18.940 traumas graves y 6.186 fracturas de cadera en ancianos.

58.816 casos de dolor torácico fueron dolores genéricos y 17.946 cardiopatías isquémicas. Dentro de este último grupo, se identificaron 2.939 infartos agudos de miocardio con elevación de ST (SCACEST), 8.611 síndromes coronarios agudos sin elevación de ST (SCASEST), 5.298 casos de otras anginas y 1.098 cardiopatías isquémicas crónicas.

Entre los ACV, se detectaron 11.268 accidentes isquémicos con o sin infarto cerebral, 7.403 accidentes isquémicos transitorios (AIT) y 2.576 hemorrágicos.
De los casos de trauma grave, 16.373 correspondieron a fracturas de húmero o fémur y 1.714 de pelvis. Entre las infecciones graves destacaron 23.773 neumonías, 3.202 síndromes de respuesta inflamatoria sistémica (SIRS) y 750 meningitis.

Como muestra la tabla 4, la edad media en varones fue de 37,61 años, el 8,66\% fueron retornos, el 9,09\% ingresaron y el $0,14 \%$ fallecieron. La edad media en mujeres (39,98 años) fue superior a la de los varones a pesar del peso de la categoría de embarazo en las urgencias de mujeres, el 8,70\% fueron retornos, el 9,39\% ingresaron y el $0,10 \%$ fallecieron. 
Tabla 4

Indicadores por sexo del total de urgencias y de los episodios de síndrome coronario agudo con elevación de ST (SCACEST). 28 Hospitales SAS 2012

\begin{tabular}{|c|c|c|c|c|c|}
\hline & & \multicolumn{2}{|c|}{ Totales } & \multicolumn{2}{|c|}{$\begin{array}{c}\text { POR } \\
\text { SCACEST }\end{array}$} \\
\hline & & Varón & Mujer & Varón & Mujer \\
\hline \multirow{5}{*}{ Características básicas } & Número total & 1.480 .056 & 1.755 .544 & 2.056 & 883 \\
\hline & Urgencias diarias & $4.043,87$ & $4.796,57$ & 5,62 & 2,41 \\
\hline & Distribución por sexo (\%) & 45,74 & 54,26 & 69,96 & 30,04 \\
\hline & Urgencias pediátricas (\%) & 25,71 & 17,93 & 0,00 & 0,00 \\
\hline & Edad media (años) & 37,61 & 39,98 & 64,74 & 75,71 \\
\hline \multirow{3}{*}{$\begin{array}{l}\text { Distribución según la evolución } \\
\text { del episodio asistencial }\end{array}$} & Pacientes que ingresan en el centro hospitalario (\%) & 9,09 & 9,39 & 89,68 & 83,72 \\
\hline & Pacientes que retornaron en menos de 72 horas (\%) & 8,66 & 8,70 & 4,57 & 4,64 \\
\hline & Fallecimientos $(\%)$ & 0,14 & 0,10 & 2,43 & 7,36 \\
\hline \multirow{4}{*}{$\begin{array}{l}\text { Indicadores temporales de } \\
\text { gestión asistencial }\end{array}$} & TPU (minutos) & 211 & 196 & 497 & 732 \\
\hline & TPU sin observación (minutos) $\dagger$ & 153 & 153 & 203 & 318 \\
\hline & Porcentaje TPU $>4 \mathrm{~h}$ sin observación $\S$ & 15,75 & 15,38 & 19,62 & 30,77 \\
\hline & Porcentaje TPU > 24h con observación $\|$ & 19,45 & 18,99 & 19,04 & 26,17 \\
\hline
\end{tabular}

TPU $=$ Tiempo medio de permanencia en urgencias en minutos, $\uparrow$ TPU sin observación $=$ Tiempo medio de permanencia en urgencias de los pacientes que no pasan por observación en minutos, $\S \%$ TPU $>4 \mathrm{H}$ (sin observación) $=\%$ de urgencias de pacientes que no pasan por observación con TPU superior a 4 horas, $\| \%$ TPU $>24 \mathrm{H}$ (con observación) $=\%$ de urgencias de pacientes que pasan por observación con TPU superior a 24 horas. 
El grupo de 15-44 años generó el mayor porcentaje de urgencias tanto en varones $(34,24 \%)$ como en mujeres $(42,09 \%)$. En mujeres el $17,67 \%$ de las urgencias en este grupo se debieron a embarazo, parto y puerperio. El mayor número de urgencias anuales por paciente se dio en los menores de 1 año $(2,09)$. Los pacientes mayores de 65 años presentaron el mayor porcentaje de ingreso $(20,55 \%$ hombres y $15,83 \%$ mujeres).

La distribución por edad y sexo varió según la patología atendida. Por ejemplo, en el ACV el porcentaje urgencias por varones y mujeres fue similar $(50,79 \%$ vs $49,21 \%)$ al igual que su mortalidad $(1,22 \%$ vs $1,08 \%)$. Sin embargo en el SCACEST el porcentaje de episodios generados por mujeres fue del 30,04\% y su mortalidad del $7,36 \%$ frente a los correspondientes $69,96 \%$ y $2,43 \%$ en varones (tabla 4). La edad media en los episodios de SCACEST en mujeres fue de 75,71 años frente a los 64,74 en varones.

La figura 1 muestra la distribución por edad y sexo de los episodios de ACV. En varones se produjo un pronunciado aumento de casos a partir de los 40 años alcanzando el máximo entre los 70 y los 79 , mientras que en mujeres la frecuencia máxima se alcanzó entre los 80 y los 89 .

Como muestra la figura 2, las horas de máxima afluencia fueron de 10:00 a 13:00 y de 16:00 a 17:00 y las horas valle de 2:00 a 8:00 de la madrugada. El patrón horario fue similar todos los días de la semana, aunque las urgencias causadas por patologías específicas presentaron distribuciones horarias propias como muestra la figura.

Los lunes tuvieron el mayor porcentaje de urgencias $(16,27 \%)$ y los sábados y domingos los menores (13,08\% y $13,58 \%$ respectivamente). Las urgencias atendidas de 0:00 a 8:00 presentaron los mayo- res porcentajes de retorno $(10,94 \%)$ y de ingreso $(14,45 \%)$.

Enero presentó el mayor porcentaje de urgencias $(9,31 \%)$ y septiembre el menor $(7,72 \%)$.

El número de urgencias totales mensuales en adultos se mantuvo prácticamente constante a lo largo del año. En los meses de invierno las urgencias se incrementaron ligeramente, sobre todo por la contribución de las pediátricas.

Se observaron distintos patrones de estacionalidad por categorías clínicas. Las lesiones e intoxicaciones, las enfermedades de la piel, genitourinarias y mentales presentaron ligeros incrementos en los meses de verano mientras que las enfermedades respiratorias y circulatorias lo hicieron en los de invierno.

Dentro de cada categoría clínica, las distintas patologías presentaron perfiles propios. Por ejemplo, en aparato respiratorio, las urgencias por reacciones alérgicas no presentaron variaciones mensuales significativas, mientras que el $76,07 \%$ de las causadas por gripe se acumularon entre enero y febrero, el $54,49 \%$ de las causadas por EPOC entre diciembre y marzo y las causadas por asma alcanzaron su máxima frecuencia relativa en primavera y la mínima en verano (figura 3 ).

Los pacientes que no pasaron por observación $(92,67 \%)$ permanecieron en urgencias 153 minutos de media (141 los no ingresados y 355 los ingresados) y un $15,55 \%$ de ellos más de 4 horas $(13,80 \%$ de los no ingresados y $44,16 \%$ de los ingresados). Los pacientes que pasaron por observación $(7,33 \%)$ permanecieron en urgencias 858 minutos de media (704 los no ingresados y 1.072 los ingresados) y un $19,23 \%$ de ellos más de 24 horas $(12,20 \%$ de los no ingresados y $29,04 \%$ de los ingresados). 
Figura 1

Urgencias según tipo de accidente cerebro-vascular (ACV), sexo y edad.

CMBD Urgencias SAS 2012

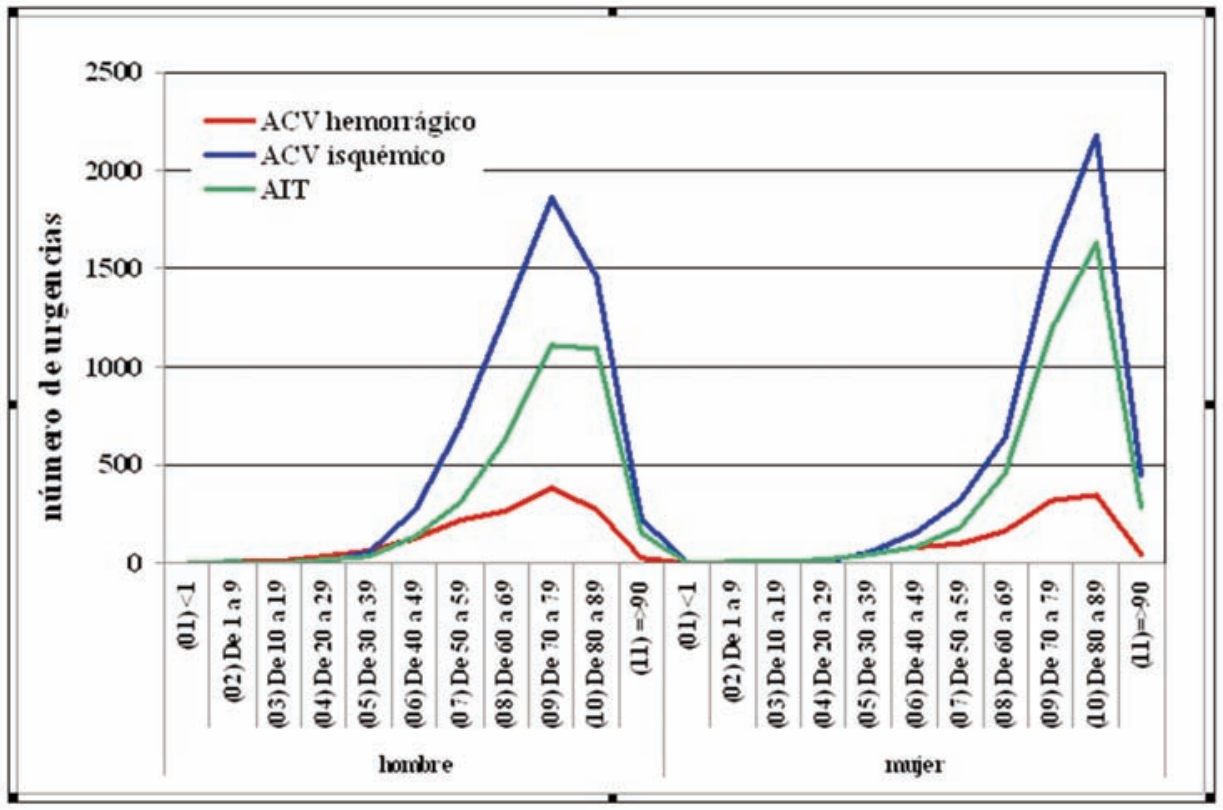

Figura 2

Porcentaje de admisiones del total de urgencias, y de las generadas por "SCACEST", "ACV" y "alcohol" por tramo horario. CMBD Urgencias SAS 2012

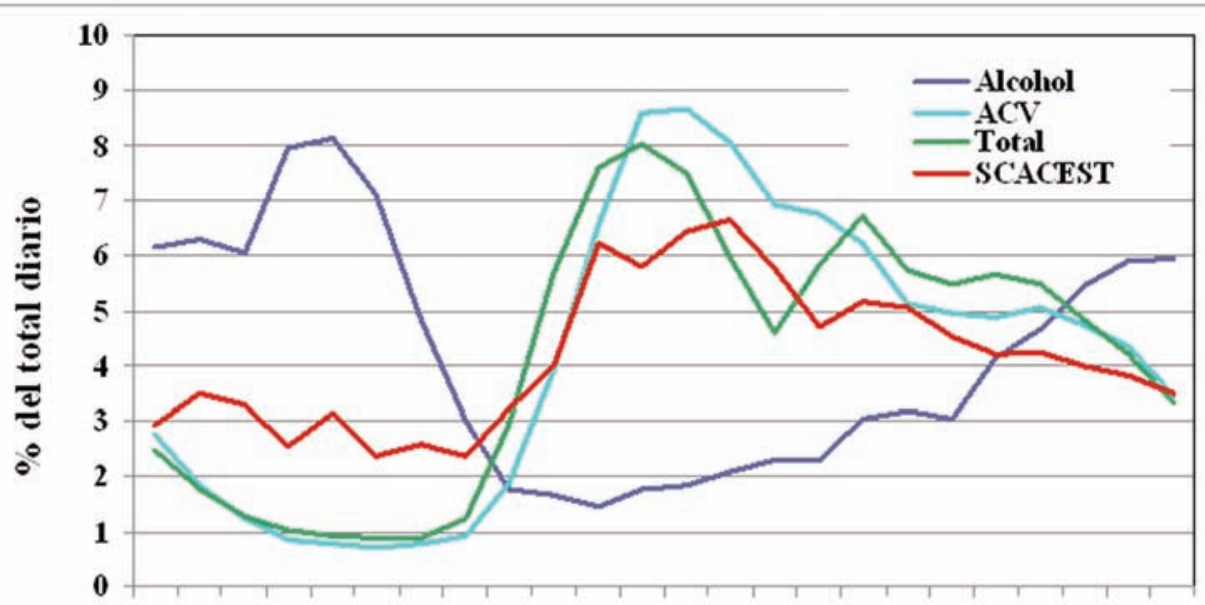

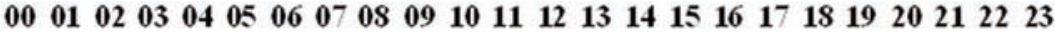

a a a a a a a a a a

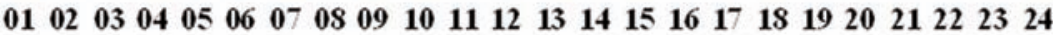


Figura 3

Porcentaje mensual de urgencias en determinados grupos de la categoría CCS “Aparato respiratorio". CMBD Urgencias SAS 2012

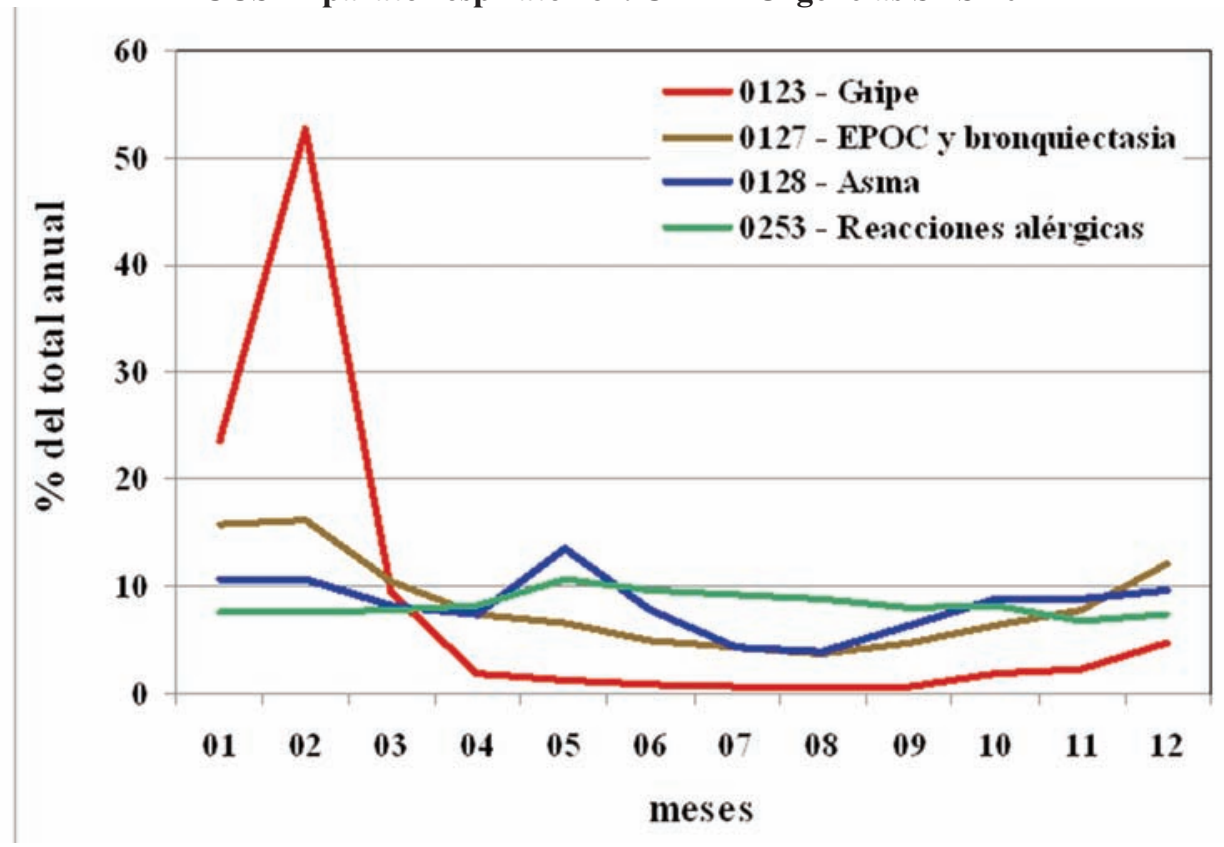

\section{DISCUSIÓN}

Durante el año 2012 cada mil habitantes adscritos a los hospitales del SAS generaron 500 urgencias hospitalarias. Una quinta parte fueron pediátricas. Un tercio de las urgencias en adultos se debieron a lesiones e intoxicaciones y enfermedades osteomusculares, mientras que en pediatría las enfermedades del aparato respiratorio y las lesiones e intoxicaciones fueron la causa de prácticamente la mitad de las urgencias. En la mayoría de las categorías clínicas de adultos predominaron las mujeres mientras que en pediatría lo hicieron los hombres. El patrón horario presentado por las urgencias fue muy estable, si bien determinadas patologías presentaron patrones horarios específicos. El porcentaje global de retornos en 72 horas fue similar en adultos y niños, manteniéndose muy estable a lo largo del periodo del que disponemos datos (2009-2012).
Ya entre enero de 1999 y marzo de 2000 , Conesa et al. ${ }^{8}$ describieron la casuística de una muestra aleatoria de 11.188 urgencias de seis hospitales catalanes y Torne et al ${ }^{9}$ analizaron, durante 3 semanas de 1999 y 2000, 67.791 urgencias atendidas en 10 hospitales y 65 centros extrahospitalarios públicos de Barcelona. En 2002 Gómez et al. ${ }^{10}$ describieron 35.731 urgencias de un hospital andorrano y en 2007, Pérez-Ciordia y Guillén ${ }^{11}$ analizaron 2.364 cuestionarios autocumplimentados durante una semana por usuarios de las urgencias en Navarra sobre sus motivos para acudir a ellas. Moreno-Millán ${ }^{12}$ et al. estudiaron la prevalencia de patologías urgentes en hospitales españoles de agudos durante 2002-2007, pero únicamente en aquellas urgencias de mayores de 44 años seguidas de ingreso. En 2010 Ansorena et al. ${ }^{13}$ comunicaron datos sobre 673.534 urgencias del Hospital de Sierrallana de Cantabria entre 1998 y 2008. En 2011, 
tras la implantación de un codificador automático, el Clinic de Barcelona presentó la clasificación de 28.609 urgencias correspondientes al $30,11 \%$ más frecuente de su casuística $^{14}$

En tres de estos estudios ${ }^{9-11}$ las lesiones y envenenamientos, los síntomas y signos mal definidos, las enfermedades respiratorias y las del aparato locomotor aparecen, como en el nuestro, entre las primeras causas de urgencias. Mayor discrepancia casuística encontramos con la del Hospital de Sierrallana, cuyas urgencias más frecuentes se debieron a patologías músculoesquelética, de piel y tejido subcutáneo y del aparato digestivo ${ }^{13}$.

En varios estudios el porcentaje de episodios de mujeres es similar al nuestro ${ }^{8,9,15}$, así como el de episodios pediátricos y la edad media de los pacientes atendidos ${ }^{8,11}$. Nuestros patrones de asistencia horaria y por días de la semana son análogos a los de Torne et al. ${ }^{9}$ y el porcentaje de ingresos similar al encontrado por Peiró et al. ${ }^{19}$.

A diferencia de los estudios mencionados, el nuestro es de base poblacional y, aunque únicamente presentamos resultados de 2012, los datos se muestran consistentes a lo largo del periodo del que se dispone de información (2009-2012).

En el caso del SAS los tiempos de asistencia y de permanencia en urgencias son muy inferiores en los niños en relación a los adultos. También lo es el porcentaje de urgencias con tiempo de permanencia superior al establecido como objetivo. La comparación de los tiempos asistenciales y de permanencia en los servicios de urgencias del SAS con los de otros Servicios Regionales u hospitales requeriría de una definición homogénea, fundamentalmente en lo referente a la identificación del momento de salida del servicio y el de hospitalización en el caso los pacientes ingresados, que pueden variar dependiendo de los distintos modelos organizativos. Igualmente, en la comparación de dichos tiempos es necesario tener en cuenta factores como la complejidad hospitalaria, la presión asistencial, el resultado de la clasificación en el triaje o las características y casuística que presentan los pacientes atendidos.

También los porcentajes de mortalidad en los servicios de urgencia, que en el caso del SAS son bajos tanto en adultos como en pediatría, pueden influenciarse por los distintos modelos organizativos y por la definición del momento de ingreso en hospitalización.

Aunque este artículo recoge una aproximación meramente descriptiva a las urgencias hospitalarias permite orientar futuros estudios analíticos en los que explorar en detalle la relación entre las distintas variables contenidas en el CMBD-U (asociaciones, distribuciones temporales, perfiles de pacientes, etc.). El CMBD-U del SSPA recoge información ininterrumpida de más de tres millones de episodios anuales codificados con CIE-9-MC y proporciona información detallada sobre la demanda en las Urgencias hospitalarias, la casuística, complejidad y procedencia de los pacientes y el tipo de asistencia que se les presta, dando respuesta a muchas de las necesidades de información de los distintos niveles de la organización sanitaria relacionados con la planificación y gestión de este ámbito.

Prevemos que la consolidación del CMBD-U y la retroalimentación a los profesionales de los servicios de urgencias corregirán en parte algunas deficiencias detectadas en este estudio, como la ausencia de texto codificable en un 3,38\% de registros o la poca especificidad de los textos diagnósticos en los pacientes fallecidos $(21,61 \%$ de las defunciones en adultos se clasifican en la categoría de códigos residuales). 
Otro aspecto mejorable de este registro es la identificación del momento de entrada de los pacientes en la consulta médica y por tanto la precisión del TPAM, que actualmente podría ser inexacto para algunos tipos de pacientes. La depuración de la Base de Datos de Usuarios del SSPA permitiría además reducir algunas inconsistencias encontradas entre las clasificaciones clínicas y la edad y sexo de los pacientes (afecciones perinatales en adultos o complicaciones del embarazo pediátricas).

También existe un margen para mejorar el Recall (sensibilidad) del codificador automático $(0,8784$; IC95\%: 0,8436-0,9100). Sin embargo, la mejora en su precisión (valor predictivo positivo) $(0,9760$; IC95\%: 0,9574$0,9900)$ apenas influiría en las clasificaciones casuísticas obtenidas.

Sobre las limitaciones metodológicas del estudio cabe mencionar que al clasificar los episodios en grupos y categorías CCS utilizando únicamente el diagnóstico principal se ignoran sus comorbilidades y complicaciones, aunque el bajo número medio de diagnósticos codificados por episodio reduce la trascendencia de esta limitación. La posibilidad de identificar varios PAI en un mismo episodio también puede provocar algunas incoherencias en el análisis, al imputarse atributos de un mismo episodio a distintos PAI simultáneamente (por ejemplo su mortalidad). La utilización de agrupadores casuísticos para clasificar unívocamente los episodios urgentes ayudaría a resolver ambas limitaciones.

A pesar de estas limitaciones, el CMBD-U puede ayudar a la ordenación y dimensionamiento de las Urgencias hospitalarias y a su coordinación con los dispositivos de atención primaria. También permite analizar la variabilidad en la práctica clínica y a establecer y monitorizar objetivos asistenciales. Facilita la identificación de casos clínicos de interés y el seguimiento de pacientes y patologías específicas. Permite estudiar aspectos relacionados con la calidad asistencial y la seguridad del paciente, como los tiempos de respuesta asis- tencial, el porcentaje de retornos o la mortalidad, generales y por causas específicas. Igualmente puede ayudar a orientar los programas docentes y generar información para sistemas de vigilancia epidemiológica o para proyectos de investigación clínica y epidemiológica.

\section{BIBLIOGRAFÍA}

1. Servicio Andaluz de Salud. Actividad Asistencial en Atención Hospitalaria Andalucía 1990. Sevilla: Consejería de Salud. Junta de Andalucía; 1992. p.302.

2. Villar F, Guerrero J, Leon C, Montaño J, Campos T. Actividad Asistencial en Atención Hospitalaria Andalucía 2000. Sevilla: Servicio Andaluz de Salud. Consejería de Salud. Junta de Andalucía; 2001. p.317.

3. García A, Montaño J, Simón I. Actividad Asistencial en Atención Hospitalaria Andalucía 2011. Sevilla: Servicio Andaluz de Salud. Sevilla: Consejería de Salud. Junta de Andalucía; 2012.p. 169.

4. Ministerio de Sanidad, Política Social e Igualdad. Información estadística de hospitales. Estadística de Centros Sanitarios de Atención Especializada. Portal Estadístico del SNS. 2011 [citado 9 de febrero de 2011]. Disponible en: http://www.msc.es/estadEstudios/estadisticas/estHospiInternado/inforAnual/homeESCRI.htm

5. Portal de la Contabilidad Analítica del SAS. [citado 11 de febrero de 2011]. Disponible en: http://inforcoan.sas.junta-andalucia.es/portal_InforCoan/Default.aspx

6. Diraya-Servicio Andaluz de Salud. [citado 15 de octubre de 2011]. Disponible en:http://www.juntadeandalucia.es/servicioandaluzdesalud/principal/documentosAcc.asp?pagina=pr_diraya

7. Friedman C, Hripcsak G. Natural language processing and its future in medicine. Acad Med. 1999;74(8):890-5.

8. Conesa A, Vilardell L, Casanellas JM, Torre P, Gelabert G, Trilla A, et al. Análisis y clasificación de las urgencias hospitalarias mediante los Ambulatory Patient Groups. Gac Sanit. 2003;17(6):447-52.

9. Torné E, Guarga A, Torras MG, Pozuelo A, Pasarin M, Borrell C. Análisis de la demanda en los servicios de urgencias de Barcelona. Aten Prim. 2003;32(07):42330 .

10. Gómez J, Pàmies S, Burgues L, Faura J. Gestión clínica de un servicio de urgencias hospitalario: indicadores de calidad, benchmarking y análisis de la casuística (case-mix). Gest hospital. 2004;15(1):3-12. 
11. Pérez-Ciordia I, Guillén F. Urgencias hospitalarias y extrahospitalarias en Navarra: Razones que las motivan. An Sist Sanit Navar. 2009;32(3):371-84.

12. Moreno E, García JM, Prieto F, Lea MC, CarbajalGuerrero J, Jiménez E, et al. Prevalencia de procesos y patologías en la atención urgente hospitalaria. An Sist Sanit Navar. 2010;33(Supl. 1):47-54.

13. Ansorena L, Pajares A, Piedra L, Matriz, M, Modino T, Crespo A. 11 años codificando urgencias en el Hospital de Sierrallana. Papeles Méd. 2010;19(1):50.

14. Conesa A, Lozano R, Casado X, Farreres R, Castellón E, Pastor X. Implantación de un sistema de codificación automática de diagnósticos en Urgencias. Libro de Ponencias y Comunicaciones. XII Congreso Nacional de Documentación Médica. Málaga: Sociedad Española de Documentación Médica; 2011. p. 196202. Disponible en: http://sedom.es/congresos/

15. Vázquez B. ¿Por qué acuden nuestros pacientes a urgencias del hospital? Aten Prim. 2000;25(3):172-5.

16. Junta de Andalucía. Consejería de Salud y Bienestar Social. Procesos Asistenciales Integrados (PAI). [citado 30 de marzo de 2013]. Disponible en: http://www.juntadeandalucia.es/salud/sites/csalud/con tenidos/Informacion General/p 3 p 3 procesos asis tenciales_integrados/listado_procesos?perfil=org

17. Junta de Andalucía. Consejería de Salud y Bienestar Social. Plan Andaluz de Urgencias y Emergencias. [citado 30 de marzo de 2013]. Disponible en: http:/www.juntadeandalucia.es/servicioandaluzdesalud/principal/documentosacc.asp?pagina=pr_ges_cal_ PlanAndUrgEmerg

18. HCUP-US Tools \& Software Page. [consultado el 18/10/2011]. Disponible en: http://www.hcupus.ahrq.gov/toolssoftware/ccs/ccs.jsp.

19. Peiró S, Librero J, Ridao M, Bernal-Delgado E, Grupo de Variaciones en la Práctica Medica en el Sistema Nacional de Salud. Variabilidad en la utilización de los servicios de urgencias hospitalarios del Sistema Nacional de Salud. Gac Sanit. 2010;24(1):06-12. 